University of New Hampshire

University of New Hampshire Scholars' Repository

Physics Scholarship

Physics

1993

\title{
COMPTEL Observations of the 1.809 MeV Gamma-Ray Line from Galactic 26Al
}

\author{
R Diehl \\ Max-Planck-Institut für extraterrestriche Physik \\ C. Dupraz \\ SRON \\ K Bennett \\ ESTEC \\ H Bloemen \\ Space Research Organization of the Netherlands \\ H deBoer \\ SRON
}

See next page for additional authors

Follow this and additional works at: https://scholars.unh.edu/physics_facpub

Part of the Astrophysics and Astronomy Commons

\section{Recommended Citation}

R. Diehl, C. Dupraz, K. Bennett, H. Bloemen, H. de Boer, W. Hermsen, G. G. Lichti, M. McConnell, D. Morris, J. Ryan, V. Schoenfelder, H. Steinle, A. W. Strong, B. N. Swanenburg, M. Varendorff, and C. Winkler, 'COMPTEL observations of the $1.809 \mathrm{MeV}$ gamma-ray line from galactic Al-26', 1993, Proc. 23rd Internat. Cosmic Ray Conf., Calgary, 1, 136.

This Conference Proceeding is brought to you for free and open access by the Physics at University of New Hampshire Scholars' Repository. It has been accepted for inclusion in Physics Scholarship by an authorized administrator of University of New Hampshire Scholars' Repository. For more information, please contact Scholarly.Communication@unh.edu. 


\section{Authors}

R Diehl, C. Dupraz, K Bennett, H Bloemen, H deBoer, W Hermsen, G G. Lichti, Mark L. McConnell, D Morris, James M. Ryan, V. Schonfelder, H Steinle, A W. Strong, B Swanenburg, M Varendorff, and C Winkler 


\section{COMPTEL Observations OF the 1.809 MeV Gamma-RAY LiNe FROM GALACTIC ${ }^{26}$ AL}

R. Diehl ${ }^{1}$, C. Dupraz ${ }^{2}$, K. Bennett ${ }^{4}$, H. Bloemen ${ }^{2}$, H. deBoer ${ }^{2}$, W. Hermsen ${ }^{2}$, G.G. Lichti $^{1}{ }^{1}$ M. McConnell ${ }^{3}$, D. Morris ${ }^{3}$, J. Ryan $^{3}$, V. Schönfelder ${ }^{1}$, H. Steinle ${ }^{1}$, A. W. Strong ${ }^{1}$, B.N. Swanenburg ${ }^{2}$, M. Varendorff ${ }^{1,3}$, C. Winkler ${ }^{4}$

1 Max Planck Institut für extraterrestrische Physik, D-8046 Garching, FRG

2 Laboratory for Space Research (SRON) Leiden, P.B. 9504 NL-2300 RA Leiden, The Netherlands

3 Institute for Studies of Earth, Oceans and Space, University of New Hampshire, Durham, NH 03824, USA

4 Astrophysics Division of ESAESTEC, NL-2200 AG Noordwijk, The Netherlands

\section{Abstract}

The COMPTEL experiment on the Compton Gamma-Ray Observatory has been demonstrated to be capable of imaging the Galaxy within its field of view of about 1 steradian in the $1.809 \mathrm{MeV}$ gamma-ray line originating from radioactive ${ }^{26} \mathrm{Al}$. The combined data from the Compton GRO sky survey in 1991/1992 have been analyzed to provide a first map of the inner Galaxy in this gamma-ray line. The $1.809 \mathrm{MeV}$ emission appears extended along the inner $70^{\circ}$ of the Galactic plane, with a relatively sharp falloff outside this regime. Possible correlations with other tracers of the candidate ${ }^{26} \mathrm{Al}$ sources are discussed.

\section{Introduction}

The $1.809 \mathrm{MeV}$ gamma-ray line originating from the decay of radioactive ${ }^{26} \mathrm{Al}(1.04$. $10^{6}$ years lifetime) had been predicted by Ramaty and Lingenfelter already in 1977 as being observable proof of recent nucleosynthesis in the Galaxy. It was detected for the first time with the HEAO-C instrument (Mahoney et al., 1984), and confirmed by many other measurements since then. The formation of ${ }^{26} \mathrm{Al}$ takes place in nucleosynthesis sites such as novae, supernovae, and in the interior of massive stars (Nofar et al. 1991, Woosley 1986, Prantzos 1993, Paulus and Forestini 1991). As the Galactic distribution of these potential ${ }^{26} \mathrm{Al}$ sources can be inferred to some extent from measurements in other spectral regimes, imaging of the $1.8 \mathrm{MeV}$ emission in the Galaxy is believed to provide the clue to the nature of the source. Various attempts to image the line emission have prevented a conclusive result due to limitations in sky exposure or instrumental capabilities, although extended source models appeared somewhat more likely.

The Compton Gamma-Ray Observatory (CGRO) with its four gamma-ray instruments (BATSE, OSSE, COMPTEL, EGRET) was successtully launched in April 1991. The COMPTEL imaging telescope has adequate sensitivity (Schönfelder et al., 1993) to provide new insight into the origin of ${ }^{26} \mathrm{Al}$. First $1.809 \mathrm{MeV}$ results from this instrument have been reported by Diehl et al. (1993a,b,c). This paper presents preliminary imaging results for the inner 2 radians of the Galactic plane in the 1.809 $\mathrm{MeV}$ gamma-ray line from a combination of all observations of the CGRO sky survey (April 1991 - November 1992). 


\section{Data Analysis}

With an instrumental energy resolution of $8.5 \%$ (FWHM) at $1.8 \mathrm{MeV}$ and a high photopeak fraction at $\mathrm{MeV}$ energies, the $1.809 \mathrm{MeV}{ }^{26} \mathrm{Al}$ gamma- ray line can be identified already in the raw data from the COMPTEL scintillation detectors. The dominant features of the instrumental background are similar for all observations, with known strong instrumental background lines at 1.46 and $2.2 \mathrm{MeV}$. Therefore, an average of several high-latitude observations provides a good first-order background model for identification of the line. The imaging analysis was performed in two different approaches:

1. Using the imaging information measured for each event, the signal from a narrow $\left(\sim 10^{\circ}\right)$ region in the sky can be selected ('software collimation'). Variation of the selection criteria can be used to scan excess $1.8 \mathrm{MeV}$ emission within the field of view. Background subtraction was performed from averages of several high-latitude observations, normalized to the instrumental $2.2 \mathrm{MeV}$ line.

2. Using the full imaging response of the telescope, a sky image can be generated from the 3-dimensional dataspace of measured scatter angle and direction. Two image-generation algorithms were applied:

- The 'Maximum Entropy' method iteratively extracts a sky image using the 3dimensional PSF by maximizing the overall image entropy until satisfactory agreement with the data is achieved.

- The 'Maximum likelihood' method scans the dataspace with the (3-dimensional) PSF, maximizing the likelihood for a hypothetical source at each scan position successively.

Detailed background modelling in the 3-dimensional data-space is achieved through different methods:

a) Data from the same observations at adjacent energy bands are smoothed.

b) Data from the same observation and energy band are filtered to extract instrumental background with minimum celestial signal suppression (Bloemen et al., 1993).

c) Observations from high Galactic latitudes in the same energy band are used.

The latter two background models provide a crosscheck on the $1.809 \mathrm{MeV}$ line versus continuum contribution.

\section{Results}

The Galactic plane has been observed during the CGRO sky survey in 23 pointings of the field of view of 1 sterad. A scan profile of excess $1.8 \mathrm{MeV}$ counts in the direction of the Galactic Center was compared to simulations of the expected longitude profiles for a point source, supernova, and nova origin, using the same 'software collimation' analysis (Diehl et al., 1993c). From this comparison, a point source alone at the Galactic center is excluded, while all other models are compatible with these data. Imaging analysis with the full instrument response in the 3-dimensional event dataspace of scatter direction and -angle provides a more detailed map of the $1.809 \mathrm{MeV}$ emission. Figure 2 presents a first maximum likelihood map from the combined observations of the sky survey for the inner Galaxy (longitude range $\pm 60^{\circ}$ ). Extending the earlier results from four observations (Diehl et al. 1993b), it is evident that combining more observations results in substantial reduction of the noise in the 
images, confirming the basic structures of early results. The $1.809 \mathrm{MeV}$ emission appears extended along the Galactic plane over a longitude range from -35 deg to 40 deg; beyond this regime, the intensity drops by about a factor 3; the inner Galaxy emission is fairly inhomogeneous with a particularly pronounced maximum close to, but not at the Galactic center itself (the statistical probability of the maximum being the Galactic Center is $<10^{-3}$, from the measured likelihood difference between the maximum of emission and $0 \% 0^{\circ}$ ). If all observations are combined and deconvolved with the 'Maximum Entropy' method, essentially the same image results, although with somewhat finer details due to the inherent deconvolution in this method (Diehl et al., 1993c).

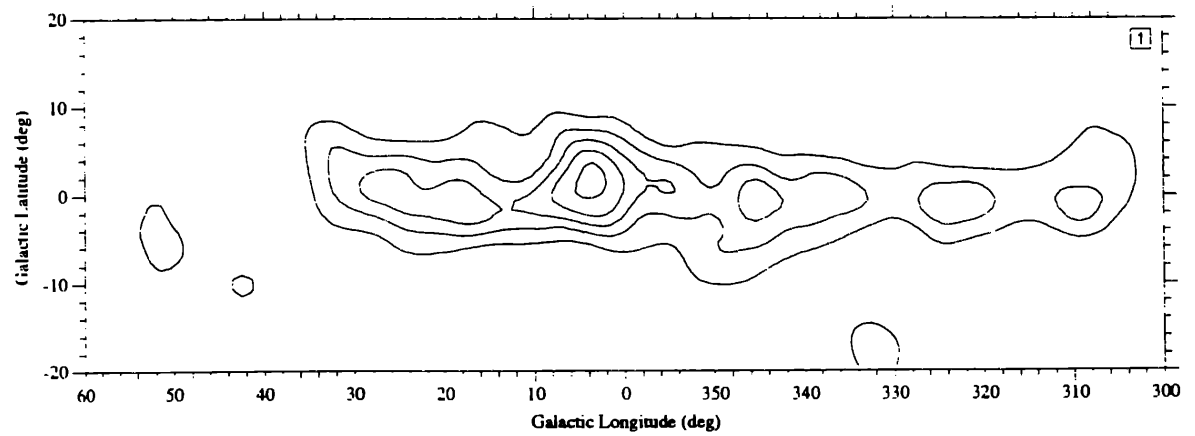

Figure 1: $\quad$ Map of likelihood values for 1.8 MeV emission along the Galactic plane. All observations of the plane from the CGRO sky survey are combined.

\section{Discussion}

${ }^{26} \mathrm{Al}$ is believed to originate in nucleosynthesis sites such as novae, supernovae type II, or massive stars. The conditions at these sites are required to be sufficiently hot and enriched in ${ }^{26} \mathrm{Al}$ seed nuclei such that the ${ }^{26} \mathrm{Al}$ production through the $\mathrm{Mg}-\mathrm{Al}$ cycle (Woosley, 1986) is effective, but on the other hand destruction of ${ }^{26} \mathrm{Al}$ due to competing neutron or proton capture reactions in such hot environments should be sufficiently small. These constraints favour non-equilibrium nuclear burning such as explosive nucleosynthesis on the surface of metal enriched O-Ne-Mg novae or in the burning shockwave of supernovae type II (core collapse supernovae). An alternative process is core nuclear burning in massive stars with convective stellar atmospheres, where the synthesized ${ }^{26} \mathrm{Al}$ is convected away from the hot inner burning region sufficiently fast. Precise yield calculations are very difficult, as the delicate balance of nuclear reactions depends critically on temperatures and convection, hence requires calculations with adequate treatment of the hydrodynamics of the nucleosynthesis region. Therefore, the question of ${ }^{26} \mathrm{Al}$ origin can be addressed most directly from Galactic mapping of the $1.8 \mathrm{MeV}$ emission, and correlation of this emission image with the Galactic distributions of the candidate sources. Due to the lifetime of ${ }^{26} \mathrm{Al}$ of $10^{6}$ years, integration of individual nucleosynthesis events over this timescale is required. If the yield in an individual event is a substantial fraction of the totally observed ${ }^{26} \mathrm{Al}$, a clumpy emission picture can be expected; on the other hand, for low individual event yields as expected for novae and massive stars in general, the emission profile should be fairly smooth. 
From the COMPTEL data, smooth source models such as the classical nova model (Higdon and Fowler, 1987) can be ruled out, as well as attribution of the observed ${ }^{26} \mathrm{Al}$ to a single pointlike source; detailed model fitting is in progress. Overlays of the $1.8 \mathrm{MeV}$ emission map with Wolf-Rayet stars in the Galaxy (from catalogue v.d. Hucht et al., 1988 indicate that these appear to trace the $1.809 \mathrm{MeV}$ emission much better than e.g. supernova remnants (see Diehl et al., 1993c). This may be interpreted as the WR stars tracing young massive stars in the Galaxy, hence young massive stars and their supernovae (type $\mathrm{Ib}$ and II) being responsible for the Galactic ${ }^{26} \mathrm{Al}$; the general SNR catalogue (Green 1988) probably contains mostly supernovae type I, which are expected to be relatively insignificant in ${ }^{26} \mathrm{Al}$ yield. Possibly, however, the observed SNR distribution is not a good tracer of the progenitors in general (van den Bergh, 1988). The observed location of the central emission peak is compatible with the peak of the Galactic gas distribution as measured in CO (Dame et al., 1987); possibly $\mathrm{CO}$ traces regions of massive star formation, hence also enhanced densities of young massive stars or supernovae type II as potential ${ }^{26} \mathrm{Al}$ sources. ROSAT maps of the diffuse Galactic X-ray continuum emission at $1.5 \mathrm{keV}$ (Snowdon, 1992) show the Galactic bulge, but also indicate more local emission that extends out of the Galactic disk; such regions of hot gas with enhanced star formation/supernova activity may be exploited to further substantiate the 'massive star' origin of ${ }^{26} \mathrm{Al}$.

\section{Conclusions}

First combined COMPTEL data from all observation periods of the Compton GammaRay Observatory sky survey yield a map of the inner region of the Galaxy in the light of the $1.809 \mathrm{MeV}$ gamma-ray line attributed to radioactive ${ }^{26} \mathrm{Al}$. The image extends over a broad longitude range. Further observations in other regimes of the Galaxy are needed to put constraints on the origin of the ${ }^{26} \mathrm{Al}$ emission. It is remarkable that the clumpy emission as seen in COMPTEL data indicates the possibility of local ${ }^{26} \mathrm{Al}$, i.e. the observed ${ }^{26} \mathrm{Al}$ is not necessarily concentrated in the vicinity of the Galactic center at distances of about $8.5 \mathrm{kpc}$. If more local ${ }^{26} \mathrm{Al}$ is observed, the ${ }^{26} \mathrm{Al}$ mass derived from the $1.8 \mathrm{MeV}$ line intensity may be well below the $1-3 \mathrm{M}_{0}$ derived from the measurements.

\section{References:}

Bloemen, H. et al., 1993, Ap.J.Suppl., to be published

Dame T.M., et al., 1987, Ap.J., 322, 706

Diehl R., et al., 1993a, Astr.\&Astrop. Suppl., 97, 1, 181

Diehl R., et al.,1993b, AIP Conf. Proc. St. Louis, in press

Diehl R., et al., 1993c, Ap.J.Suppl., to be published

Paulus, G., and Forestini, M., 1991, AIP Conf.Proc. 232, 157

Green, D.A., 1988, ASS, 148, 3

Higdon, J.C., and Fowler, W.A., 1987, Ap.J. 317, 710

Nofar, I., Shaviv, G., and Starrfield, S., 1991, Ap.J. 369,440

Prantzos, N., 1993, Ap.J. Lett, in press

Purcell, W.R., et al., 1988, AIP Conf.Proc. 170, 181

Ramaty, R., and Lingenfelter, R.E., 1977, Ap.J. 213, L5

Schönfelder, V. et al., 1993, Ap.J. Suppl., in press

Snowdon, S.L., 1992, private communication

Teegarden, B., et al., 1991, AIP Conf.Proc. 232, 116

van den Bergh, S., 1988, in: "Supernova Shells and their Birtth Events", Ed. W. Kundt, p 44

van der Hucht, K., 1988, Astr.\&Astroph. 199, 217-234

von Ballmoos, P., Diehl, R., and Schönfelder, V., 1987, Ap.J. 318, 654

Walter, R., and Maeder, A., 1989, Astr\&Astrop., 218, 123

Woosley, S.E., 1986, in "Nucleosynthesis and Chem. Evolution", өd. B. Hauck and A. Maeder, p 1 